\title{
A Random-Object-Kinematogram Plugin for Web-Based Research: Implementing Oriented Objects Enables Varying Coherence Levels and Stimulus Congruency Levels
}

\author{
Younes Strittmatter*, Markus Wolfgang Hermann Spitzer*, Andrea Kiesel \\ University of Freiburg, Department of Cognitive Psychology \\ *Equal Contribution
}

Corresponding Author: markus.spitzer@psychologie.uni-freiburg.de

\begin{abstract}
Author Note
We thank Janina Janz and Jessica Helm for helpful comments on an earlier version of the manuscript. Raw data and commented analysis scripts are available via the Open Science Framework at https://osf.io/crzsy/. The ROK plugin is fully available, documented, and free to use on https://github.com/younesStrittmatter/ROK-plugin.
\end{abstract}




\begin{abstract}
One of the recent major advances in cognitive psychology research has been the option of web-based in addition to lab-based experimental research. This option fosters experimental research by increasing the pace and size of collecting data sets. Importantly, web-based research profits heavily from integrating tasks that are frequently applied in cognitive psychology into open access software. For instance, an open access random-dot kinematogram (RDK) plugin has recently been integrated into the jsPsych software for web-based research. This plugin allows researchers to implement experimental tasks with varying coherence levels (with that varying task difficulty) of moving dots or varying signal to noise ratios of colored dots. Here, we introduce the random-object kinematogram (ROK) plugin for the jsPsych software which, among other new features, enables researchers to include oriented objects (e.g., triangles or arrows) instead of dots as stimuli. This permits experiments with feature congruency (e.g., upwards moving triangles pointing upwards) or incongruency (e.g., upwards moving triangles pointing downwards), allowing to induce gradual degrees of stimulus interference, in addition to gradual degrees of task difficulty. We elaborate on possible set-ups with this plugin in two experiments examining participants' RTs and error rates on different combinations of coherence and congruency levels. Results showed increased RTs and error rates on trials with lower coherence percentages, and on trials with lower congruency levels. We discuss other new features of the ROK plugin and conclude that the possibility of gradually varying the coherence level and congruency level independently from each other, offers novel possibilities when conducting web-based experiments.
\end{abstract}




\section{A Random-Object-Kinematogram Plugin for Web-Based Research: Implementing Oriented Objects Enables Varying Coherence Levels and Stimulus Congruency Levels}

\section{Introduction}

The number of online experiments conducted throughout the past decade has increased massively in several academic fields, such as cognitive psychology, developmental psychology, and social psychology, with web-based experiments reveiling similar data quality, but faster data collection, as lab-based experiments (Buhrmester, Kwang, \& Gosling, 2011; Crump, McDonnell, \& Gureckis, 2013; Ramsey, Thompson, McKenzie, \& Rosenbaum, 2016). A major milestone to foster web-based research was the introduction of open access software such as jsPych (de Leeuw, 2015) and the implementation of plugins for experimental set-ups that can easily be applied with this software. For instance, the random dot kinematogram (RDK) plugin (see Rajananda, Lau, \& Odegaard, 2018) allows researchers to easily apply RDK stimulus displays (Benetti et al., 2021; Britten, Shadlen, Newsome, \& Movshon, 1992; Danielmeier, Eichele, Forstmann, Tittgemeyer, \& Ullsperger, 2011; Guterstam \& Graziano, 2020; Guterstam, Wilterson, Wachtell, \& Graziano, 2020; Kang et al., 2021; Kayser, Erickson, Buchsbaum, \& D’Esposito, 2010, Krueger et al., 2017; Mante, Sussillo, Shenoy, \& Newsome, 2013; Marques et al., 2018; Purcell \& Kiani, 2016; Ritz \& Shenhav, 2019, Shadlen, Britten, Newsome, \& Movshon, 1996, Shenhav, Straccia, Musslick, Botvinick, \& Cohen, 2018; Spitzer, Kiesel, \& Dignath, 2021; Spitzer, Musslick, Shvartsman, Shenhav, \& Cohen, 2019; von Lautz, Herding, \& Blankenburg, 2019) in order to implement the random-dot motion task in online experiments (for example see: Bhui, 2019; Krzeminski \& Zhang, 2021; Musslick, Bizyaeva, Agaron, Leonard, \& Cohen, 2019).

The RDK stimulus displays contain varying numbers of dots that move in a target, opposing to the target, or random direction and participants are instructed to detect in which specific direction the majority of dots move (i.e., the coherent direction). One feature of this task is that the difficulty of the task can be adjusted gradually by decreasing the motion coherence (to make the task harder) or increasing 
the motion coherence (to make the task easier). In addition to the motion identification task, dots may be presented in two different colors, enabling a color discrimination task. For this color task, participants are instructed to detect the dominant of two different colors while the ratio of colors can be varied to allow different task difficulties (Krueger et al., 2017; Musslick et al., 2019; Shenhav et al., 2018; Spitzer et al., 2019; Steyvers, Hawkins, Karayanidis, \& Brown, 2019).

Here, we introduce an advanced version of the RDK plugin - the random object kinematogram (ROK) plugin. The ROK plugin incorporates the functionalities of the RDK-plugin but additionally enables researchers to (a) upload visual objects instead of dots as stimuli, (b) implement multiple ROK stimulus displays at the same time, and (c) integrate background pictures (see Table 1 for an overview of ROK plugin parameter settings). This set of new functionalities allows more flexibility on the stimulus presentation and thus expands the possibilities of experimental tasks one can design using the ROK plugin which we describe in the following sections. The ROK plugin is fully available and free to use on https://github.com/younesStrittmatter/ROK-plugin. A documentation as well as a demonstration of the ROK plugin is available on this github link, but a short description of how to use the plugin shall briefly be described. The jsPsych JavaScript library (https://www.jspsych.org/) is a prerequisite to run the plugin. If jspsych is installed, copy the file jspsych-rok.js (located in the plugins folder of the repositiry) into the plugins-folder of the jsPsych library. To use the stimuli provided with this plugin, copy the res-folder into your experiments project folder. See the documentary (docs) folder or the example folder for further instructions. You can use the examples folder (alongside the jsPsych libary) as template to run your own experiments.

The major novelty of the ROK plugin is the implementation of object stimuli instead of dot stimuli. Object stimuli can include features which may lead to interference on the stimulus level. For example, imagine a set of arrow stimuli, instead of dot stimuli, presented with the ROK plugin (see Figure 1). Moving arrows now expand the feature space of the ROK, with a proportion of these arrows moving in a 
coherent direction, while the remaining arrows move in random direction and, at the same time, a proportion of these arrows orienting in a coherent direction, while the remaining arrows are oriented in random direction. With this setup, one can instruct participants to respond to one of the two stimulus dimensions: moving direction or orientation direction. If objects move and are oriented in only two directions (e.g., up and down) and participants are instructed to detect the dominant movement direction, moving arrows can be oriented in the same direction, which we refer to as a congruency level of $100 \%$, or be directed in opposing directions, which we refer to as a congruency level of $0 \%$. But also any congruency level between $100 \%$ and $0 \%$ is possible. For example, the congruency level of the two stimuli can be $50 \%$. In this case, if participants are instructed to respond to the motion task, with the two directions up and down, half of the objects would be oriented upwards, while the other half would be oriented downwards. In sum, with this setup of two stimulus dimensions (moving direction and orientation direction) the ROK plugin enables flexible research with a task of varying difficulty levels, by changing the coherence of the instructed dimension, and varying congruency levels, by changing the coherence of the irrelevant dimension .

Another new feature of the ROK plugin is that researchers can present multiple layers of ROK stimuli at once in one trial (see Figure 1) and the described features of coherence and congruency level can be manipulated for each stimulus layer, respectively. The objects of each layer can be presented "on top of each other", in a defined order, or in randomised order. Here, we labeled the stimulus layer participants are instructed to respond to as the target layer and labeled the stimulus layer with distractor stimuli as distractor layer. For example, if participants are instructed to respond to upwards or downwards moving and oriented arrows, then the target layer would be the random-arrow kinematogram. If now a second stimulus layer (e.g., a random-square kinematogram) would simultaneously be presented but participants are instructed to disregard these stimuli, this layer would then be denoted as the distractor layer.

Finally, users may implement background pictures for their ROKs. This allows researchers to implement the ROK stimuli in different contexts, depending on the 
background picture.

\section{The Present Experiments}

Here, we applied the ROK plugin for the jsPsych software in two experiments and demonstrated some of the possible usage variants. Therefore, we first built the ROK plugin (for an overview of the parameter settings of the ROK plugin, see Table 1 . see Table 2 for an overview of which parameters additionally get generated) and tested the data collection quality (see Figure 2). Please note that the ROK plugin interfaces with the jspsych-resize plugin to allow for standardized displays. We then conducted Experiment 1, which included isosceles triangles as stimuli objects and with two tasks, (1) detecting the majority of upwards or downwards moving triangles, or (2) identifying the majority of upwards or downwards oriented triangles. With these two tasks, we applied different coherence and congruency levels and asked whether lower coherence and congruency levels would increase RTs and error rates and tested whether the two factors (coherence and congruency level) interacted with each other (Analysis 1a \& 1b). Moreover, we tested whether the effect of coherence and congruency level differed between tasks. Finally, we investigated whether the addition of a distraction layer would increase RTs and error rates compared to no distraction layer while we kept the coherence and congruency level constant (Analysis 1c \& 1d). Each of these analyses was then replicated with leafs as stimuli objects and on a naturalistic background in Analysis 2a-d.

In both experiments, we expected RTs and error rates to increase with decreasing coherence levels and with decreasing congruency levels. We expected increased RTs and error rates when a distractor layer is added compared to non-distractor trials. Finally, we had no prior hypothesis which of the two tasks (movement vs. orientation) was more difficult, in terms of faster or slower responses and higher or lower error rates on one of the two tasks. 


\section{Experiment 1}

Experiment 1 was conducted to examine whether the possibility to gradually adjust the coherence and the congruency level of the task affected RTs and error rates. We applied purple isosceles triangles moving and being oriented upwards or downwards as the target stimulus layer. Participants were instructed to either respond to the motion direction or the orientation direction of the triangles throughout 10 blocks. On the first five blocks participants were instructed to respond to one of the two tasks while they were instructed to respond to the other task on the second half of the blocks. The task order was counterbalanced across participants. On block 2-5, participants responded to one of the two tasks, with all possible combinations (12 combinations) of three coherence levels $(90 \%, 75 \%$, and $60 \%)$ and four congruency levels $(100 \%, 75 \%$, 25\%, and 0\%). On blocks 7-10, participants responded to the other task and the same coherence $(90 \%, 75 \%$, and $60 \%)$ and congruency level $(100 \%, 75 \%, 25 \%$, and $0 \%)$ combinations. This change of task instruction on blocks 7-10 may have led to another level of interference, namely response interference, as participants learnt how to respond to the now irrelevant task in the first half of the experiment. We added this task order factor (labeled as order) in our analyses to investigate whether the interference effect differed between the first and second half of the experiment. In addition, we asked if the coherence and congruency level effect was different between the two tasks. More precisely, the first two analyses asked whether RTs (Analysis 1a) and error rates (Analysis 1b) depended on coherence, congruency level, order, and task, including all main effects and all possible interactions between these four factors.

On block 1 and block 6 , we additionally collected data on different trials with fixed coherence and congruency levels but applied a second distractor stimulus layer. Specifically, the coherence level was set to $65 \%$ and the congruency level to $50 \%$. In addition in $10 \%$ of the trials, a distractor stimulus layer was added. We asked whether RTs (Analysis 1c) and error rates (Analysis 1d) increased on trials which included a distractor stimulus layer compared to trials with no distractor stimulus layer, and whether this effect differed between tasks. Squares, points, and randomly oriented 
triangles, colored in turquoise or yellow, served as distractor stimuli layer, while the target layer was the same as in the other blocks.

Prior to any analyses, we examined the quality of the data collection by collecting the frame rate for each response. The ROK plugin automatically collects data of the average frame rate for a trial (see Table 2 for all parameters the ROK plugin collects in addition to the parameters described in Table 11. We plotted the average frames per second $(\mathrm{Hz})$ of trial responses per participant and for each browser respectively in Figure 2, Please note that we merged the data for this plot from Experiment 1 and Experiment 2 to increase the number of participants per browser.

\section{Method}

\section{Participants}

Thirty-four participants (13 females; 21 males; $M_{\text {age }}=27.73 ; S D_{\text {age }}=5.42$ ) were recruited with Prolific and payed $£ 2.5$ (\$3.5) for their participation (max. 30 minutes). All participants provided informed consent before the start of the experiment and were told that they could stop the experiment at any given point. The sample size was based on a power analysis which expected a medium effect of the main effect of congruency level with an effect size of $d=.5$ and a power of $80 \%$ (Faul, Erdfelder, Lang, \& Buchner, 2007). Participants were only included in the final data analysis if they responded with an accuracy above $60 \%$.

\section{Stimuli}

Stimuli were presented with the jsPsych software (de Leeuw, 2015). A total of 600 purple isosceles triangles presented on black background comprised a ROK stimulus display in this experiment (see Figure 1 for a stimulus example). Objects moved and were oriented in the same two directions (up or down); yet, please note that any angle of direction could be set in the ROK plugin (for ROK plugin details see Table 1). The object size was set to $1 \%$ of aperture width and the motion speed set to $3 \%$ of aperture width per second. 
Only in block 1 and block 6, a second layer which served as a distractor layer was implemented on $10 \%$ of the trials (drawn randomly). This distractor layer comprised 70 squares, points, or randomly oriented triangles colored in turquoise and yellow with an object size of $1 \%$ of aperture width and a motion speed of $3 \%$ of aperture width per second.

\section{Procedure}

Each trial included a fixation cross presented for 500ms, followed by a ROK stimulus which was presented for up to 2000ms. Participants were instructed to respond to the motion direction or the orientation direction of the ROK stimulus by responding with the key press 'F' and 'J'. Half of the participants were instructed to press 'F' for mostly upwards moving objects and mostly upwards oriented objects, and 'J' for mostly downwards moving objects and mostly downwards oriented objects. The instructions reversed for the other half of participants.

At the beginning of the experiment, participants were provided with instructions and 16 training trials. Participants responded to a total of ten blocks each of which included 72 trials. Participants responded to one of the two tasks in blocks 1-5 and then responded to the other task on blocks 6-10. The task order was counterbalanced across participants.

During training trials and after accurate responses, the word 'CORRECT' colored in green ink was presented as feedback on the screen after each trial. After accurate responses in experimental trials no such feedback was given. After an incorrect response, the word 'FALSE' colored in red ink was presented as feedback on the screen after each trial (i.e., on training trials and on trials in block 1-10). When the participants didn't respond within $2000 \mathrm{~ms}$, the words 'TOO SLOW' appeared in red ink (on training trials and on trials in block 1-10).

Block 1 and 6 addressed a different research question and comprised different analyses than blocks 2-5 and 7-10. The differences between these blocks are described in detail in the following two sections.

The first and sixth block included the target stimulus layer of purple triangles 
and a distractor stimulus layer with points, squares and randomly oriented triangles colored in turquoise and yellow which appeared with a probability of $10 \%$ of the trials. On these two blocks the coherence was set to $65 \%$, indicating that $65 \%$ of the objects moved (or oriented) upwards or downwards while $35 \%$ of the objects moved (or were oriented) in the opposite direction. The congruency level was set to $50 \%$, indicating that the same amount of irrelevant stimuli moved (or were oriented) up and down.

On blocks 2-5 and block 7-10, only the target layer was used as ROK stimuli. The coherence of the task varied between the three levels $90 \%, 75 \%$, and $60 \%$ and the congruency level varied between the four levels 100\%, 75\%, 25\%, and 0\%. Each of the 12 coherence-congruency level combinations was applied 6 times within a block, respectively, summing up to a total of 72 trials per block. The coherence indicated the proportion of upwards and downwards moving (or were oriented) objects. For instance, $90 \%$ coherence indicated that $90 \%$ of the objects moved (or were oriented) in the target direction, while $10 \%$ of the objects moved (or were oriented) towards the non-target direction. The congruency level expressed the proportion of congruent to incongruent objects with 100\% indicating only congruent objects and $0 \%$ indicating only incongruent objects. For example, for the motion task, a congruency level of $100 \%$ indicated that all objects were oriented towards the target direction, while a congruency level of $0 \%$ indicated that all objects were oriented towards the opposite direction of the target direction. In other words, $0 \%$ congruency indicated $100 \%$ incongruency. For the orientation task, a congruency level of e.g. $75 \%$ indicated that 450 (of the 600 ) objects moved towards the target direction, while a congruency level of $25 \%$ indicated that 150 (of the 600) objects moved towards the opposite direction of the target direction Thus, the $25 \%$ congruency level expressed $75 \%$ incongruency.

\section{Independent and Dependent Variables}

We conducted four separate analyses. Two only included blocks 2-5 and blocks 7-10 and investigated the effect of coherence and congruency level on RTs (Analyses 1a) and error rates (Analyses 1b). Another two analyses only included block 1 and 6 and investigated the effect of distractor stimuli on RTs (Analyses 1c) and error rates 
(Analyses 1d). The first two analyses comprised a total of four independent variables (coherence, congruncy level, order and task). A continuous coherence variable included three coherence levels $(90 \%, 75 \%$, and $60 \%)$, indicating the percentage of objects moving in the target direction. A continuous congruency level variable, included four congruency levels $(100 \%, 75 \%, 25 \%$, and $0 \%)$, indicating the percentage of objects of the irrelevant feature moving or orienting into the coherent task direction. A categorical order variable indicated whether responses were made in the first or second half of the experiment, to investigate whether the effect of having responded to the other task on the first half of the experiment, induced an additional response congruency effect, on top of the stimulus congruency effect, and thus increased the effect of congruency level on the second half of the experiment. A categorical task variable indicated the current task.

Analyses 1c and 1d comprised two independent variables only: a categorical task variable indicating the current target task and a categorical distractor variable, indicating whether a distractor layer was absent or present. As described above, the coherence and congruency level were fixed to a specific value $(65 \%$ coherence and $50 \%$ congruency level) on trials of block 1 and block 6 and thus, coherence and congruency level was not included in Analysis 1c and 1d. Dependent variables on all four analyses comprised participants' RTs (Analyses 1a \& c) and error rates (Analyses 1b \& d) on stimulus responses, respectively.

\section{Data Analysis}

The statistical analysis was conducted with $\mathrm{R}$ ( $\mathrm{R}$ Core Team, 2013). Linear mixed models were conducted with the lmerTest package (Kuznetsova, Brockhoff, \& Christensen, 2017) and logistic mixed models were conducted with the lme4 package (Bates, Mächler, Bolker, \& Walker, 2014). Plots and tables were generated with the sjPlot package (Lüdecke, 2020). We used linear mixed models instead of analysis of variances (ANOVAs) since linear mixed models are less prone to inflated Type 1 errors (Judd, Westfall, \& Kenny, 2017; Quené, 2008; Quené \& Van Den Bergh, 2004).

In Analysis 1a and 1b, we analyzed the blocks which did not include distractor 
trials (blocks 2-5 \& blocks 7-10) and investigated RTs and error rates as a function of coherence, congruency level, order, and task, including all main effects and interaction effects. We expected slower responses as well as higher error rates on lower coherence levels and on more incongruent, compared to more congruent, trials. In addition, we asked whether response congruency further interacted with stimulus congruency, leading to a larger congruency effect, in trials with stimulus congruency and response congruency (second half) compared to trials where only stimulus congruency was manipulated (first half). We had no expectations on the effect of task and on the interactions between the four variables.

Random effects were selected based on the least complex random effect structure which accounted for most of the random effect variance (see Bates, Kliegl, Vasishth, \& Baayen, 2015). In detail, we carried out a principal component analysis on the random effects with all main effects as random slopes and a random intercept for participants. We then assessed whether the number of random effects addressed all principal components, or whether too many random effects (random slopes in specific) were included in the model. If principal components explained close to zero variance $(<$ 0.000), we excluded random slope effects explaining the least variance and fitted the model again. We followed this procedure until each principal component of the random effect structure explained 0.001 or more variance. Importantly, fixed effect results were only examined for the finally selected model. We report which of the random slope effects were included in the winning model. Please note that a random intercept for participants was always included in the model to account for individual differences on the dependent variable.

In Analysis 1c and d, we analyzed the first and sixth block of the experiment to investigate RTs and error rates as a function of distraction and task. We expected slower RTs and higher error rates on trials which included a distractor layer compared to trials that did not include distractors. Here, random effects comprised a random intercept for each participant. No random slopes were included since the model did not account for more variance when including random slopes (Bates et al., 2015). 


\section{Results}

We report the results of all main effects and all significant interactions. The complete results of each analysis is provided in tables.

\section{Exclusion Criteria}

The following exclusion criteria were applied. We excluded all trials with an RT below $200 \mathrm{~ms}$ and with no responses within 2000ms (2.77\%). For RT analyses, all incorrect responses and all responses following incorrect responses (38.70\%) were excluded. Finally, two participants were excluded prior to the data analysis due to an accuracy rate below $60 \%$.

\section{Analysis 1a: RTs as a Function of Coherence, Congruency Level, Order, and Task.}

RTs as a function of coherence and congruency level are depicted in Figure 3. All regression results are listed in Table 3. Random effects included the variables coherence, congruency level, and task, as a random slope to account for individual differences on these three variables. The order term was not included as the model did not account for more variance when including order as a random slope and we sought to apply the least complex model according to Bates et al. (2015).

The main effect of coherence was significant $(b=-433.96 ; t=-7.22 ; p=<.001)$, with faster responses on higher coherence levels. The main effect of congruency level was not significant $(b=-51.26 ; t=-1.26 ; p=.208)$. The main effect of order was not significant $(b=-47.86 ; t=-1.59 ; p=.113)$. The main effect of task was significant $(b$ $=85.49 ; t=2.83 ; p=.005)$, with slower responses on the orientation task compared to the motion task. The interaction between coherence and task was significant $(b=$ $-135.43 ; t=-3.96 ; p<.001)$, with a steeper increase in RTs with lower coherence levels on the orientation task compared to the motion task. The interaction between congruency level and task was significant $(b=88.95 ; t=2.21 ; p=.027)$, with an increased congruency effect for the motion task compared to the orientation task. None of the other interactions were significant. 


\section{Analysis 1b: Error Rates as a Function of Coherence, Congruency Level, Order, and Task.}

Error rates as a function of coherence and congruency level are depicted in Figure 3. All error rate results are listed in Table 4. Random effects included the variables congruency level, and task, as a random slope to account for individual differences on these two variables. This was the least complex random effect structure to account for most of the variance of the model.

The main effect of coherence was significant $(b=-5.95 ; z=-23.72 ; p<.001)$, with less erroneous responses on higher coherence levels. The main effect of congruency level was significant $(b=-1.11 ; z=-3.50 ; p<.001)$, with more erroneous responses on trials with higher incongruency levels. The main effect of order was not significant $(b=$ $0.10 ; z=0.52 ; p=.603)$. The main effect of task was not significant $(b=0.32 ; z=$ $1.72 ; p=.085)$. The interaction of coherence and task was significant $(b=-0.60 ; z=$ $-2.41 ; p=.016)$, with a steeper increase in error rates with lower coherence levels on the orientation task compared to the motion task. The interaction between coherence, order, and task was significant $(b=0.51 ; z=2.03 ; p=.042)$, because the increase in error rates on lower coherence levels was steeper for the orientation task than the motion task on the first half of the experiment, but steeper for the motion task than the orientation task in the second half of the experiment. But please note that the robustness of this three-way interaction has to be interpreted with caution, as our sample size was rather small.

\section{Analysis 1c: RTs as a Function of Distraction}

RTs as a function of distractor layer and task are shown in Table 5. The main effect of distraction was significant $(b=-94.74 ; t=-8.48 ; p<.001)$, with faster responses on non-distractor trials compared to distractor trials. The main effect of task was significant $(b=-23.34 ; t=-2.07 ; p=.039)$, with slower responses on the orientation task than the motion task. The interaction between distractor and task was significant $(b=39.00 ; t=3.49 ; p<.001)$, with slower RTs on the motion task than the 
orientation task on distractor trials, but faster responses on the motion task than the orientation task on no distractor trials.

\section{Analysis 1d: Error Rates as a Function of Distraction}

Error rates as a function of distractor layer and task are shown in Table 6. The main effect of distraction was not significant $(b=-0.07 ; z=-1.24 ; p=.215)$. The main effect of task was significant $(b=-0.11 ; z=-2.08 ; p=.038)$ with more errors on the motion task compared to the orientation task. The interaction of distractor and task was significant $(b=0.13 ; z=2.47 ; p=.014)$ with more errors on the motion task, compared to the orientation task, on distractor trials, but less errors on the motion task, compared to the orientation task, on non-distractor trials.

\section{Discussion}

The purpose of Experiment 1 was to apply two of the new features of the ROK plugin for the jsPsych software: (a) implementing objects instead of dots and (b) applying two ROK layers at the same time. To address the possibility of implementing objects instead of dots to enable varying degrees of stimulus interference, we investigated the effect of different combinations of coherence and congruency levels on RTs and error rates. Results revealed that RTs and error rates depended on the coherence and congruency level. With respect to the main effect of coherence, the results revealed slower and more erroneous responses on lower coherence levels. These results suggest that participants' evidence accumulation took more time and was more error-prone on trials with lower signal to noise ratio levels. These results were in line with our predictions and with previous observations (e.g., Purcell \& Kiani, 2016 ; Shenhav et al., 2018; Spitzer et al., 2019). Regarding the main effect of congruency level, participants revealed a significant effect of congruency level on error rates with more errors on trials with increasingly incongruent stimuli. Please note that the RT result on congruency level was not significant, but the descriptive pattern of this result was in line with the error rate result and does not speak in favor of a speed-accuracy trade-off (see Figure 3). To the best of our knowledge, these coherence and congruency 
level results are the first ones to show that different degrees of stimulus interference on the random-object motion task effected participants' performance significantly over and beyond the effect of different coherence levels.

We further investigated whether the task order influenced participants RTs or error rates. The motivation behind this analysis was that participants might have learnt on how to respond to the first task on block 1-5 and that this learnt response mapping induced a response congruency effect during the second half of the experiment (namely block 6-10). In this case, the effect of congruency level would be larger on the second half of the experiment compared to the first half of the experiment. However, the interaction of congruency level and order was not significant and thus did not provide evidence for an additional effect of response congruency on top of the stimulus congruency effect in the second half of the experiment. But please note that the absence of proof is not proof of absence.

In addition to these effects, we asked whether the effect of coherence, congruency level, and order further differed between the two tasks and whether one of the two tasks was easier than the other. Results revealed that the orientation task was more difficult than the motion task, as indicated by longer RTs and higher error rates on the orientation task than the motion task. The coherence variable interacted with task, showing a steeper increase in RT and error rates with lower coherence levels on the orientation task, than the motion task. In addition, we observed a significant interaction of congruency and task for RTs (but not for error rates), revealing an increased congruency effect for the motion task than the orientation task. Interestingly, the coherence and congruency variables did not interact with each other. Again, the absence of this interaction does not provide evidence whether the effects of these two variables influenced participants orthogonally. However, the descriptive pattern of these results do not indicate an interaction effect of these two variables.

In two other separate analyses we investigated whether the implementation of a distractor stimulus layer would effect RTs and error rates. Results supported our hypothesis with slower responses on distractor trials compared to non-distractor trials. 
This effect was not significant in error rates, but the result pattern descriptively points in the same direction. Besides, participants were slower and made more errors on the orientation task than the motion task. However, this main effect was driven by an increased difference between the two tasks on distractor trials.

In sum, Experiment 1 tested two new features of the ROK plugin: implementing objects instead of dots and applying two ROK layers at the same time. The implementation of objects instead of dots enables the integration of another feature, which, in this experiment, induced different degrees of stimulus congruency affecting participants' performance. Finally, results provide evidence that applying a distractor stimulus layer in addition to the target stimulus layer increased participants' RTs and error rates. In the following experiment, we asked whether the result pattern observed in Experiment 1 could be replicated with an experiment using more aesthetic stimuli and a background picture.

\section{Experiment 2}

Experiment 2 was conducted to investigate whether results from Experiment 1 would replicate when objects changed to leaves instead of triangles and with a naturalistic background. The procedure of this experiment was the same as the one of Experiment 1, except the change in objects and the inclusion of a background (see Figure 1 for a ROK stimulus layer example).

\section{Method}

\section{Participants}

As in Experiment 1, thirty-four participants (13 females; 21 males; $M_{\text {age }}=29.29$; $\left.S D_{\text {age }}=6.73\right)$ were recruited with Prolific and payed $£ 2.5$ (\$3.5) for their participation (30 minutes). All participants agreed with the informed consent provided before the start of the experiment and were told that they could stop the experiment at any given time. The sample size was based on the same criteria as in Experiment 1. Participants were only included in the final data analysis if they responded with an accuracy above 
$60 \%$.

\section{Stimuli, Procedure, Variables and Data Analysis}

Despite the change in stimuli and the addition of a background, the procedure, independent and dependent variables, and statistical analyses were the same as in Experiment 1. The random effect structure was kept the same in each analysis as in Experiment 1. All principal component analyses revealed that the random effect structure was not too complex with the proportion of variance explained by each principal component larger than zero.

\section{Results}

We report all main effects and significant interactions in the text below. The results of each individual analysis are listed in tables.

\section{Exclusion Criteria}

The following exclusion criteria were applied. We excluded all trials with an RT below 200ms and with no responses within 2000ms (1.77\%). For RT analyses, all incorrect responses and all responses following incorrect responses $(36.08 \%)$ were excluded. Finally, one participant was excluded prior to the data analysis due to an accuracy rate below $60 \%$.

\section{Analysis 2a: RTs as a Function of Coherence, Congruency Level, Order and Task.}

RTs as a function of coherence and congruency level are depicted in Figure 3, All regression results are listed in Table 3 . The random effect structure was the same as in Experiment 1.

As in Experiment 1, the main effect of coherence was significant $(b=-412.69 ; t$ $=-8.62 ; p<.001)$, with slower responses on lower coherence levels. The main effect of congruency level was significant $(b=-95.76 ; t=-2.64 ; p=.008)$, with slower responses on trials with higher incongruency levels. The main effect of order was not significant ( $b$ 
$=2.17 ; t=0.08 ; p=.938)$. The main effect of task was not significant $(b=-21.19 ; t=$ $0.75 ; p=.451)$. None of the interactions were significant.

\section{Analysis 2b: Error Rates as a Function of Coherence, Congruency Level, Order, and Task.}

Error rates as a function of coherence and congruency level are depicted in Figure 3. All regression results are listed in Table 4. The random effect structure was the same as in Experiment 1.

The main effect of coherence was significant $(b=-5.93 ; z=-22.92 ; p<.001)$, with more erroneous responses on lower coherence levels. The main effect of congruency was not significant $(b=-0.43 ; z=-1.29 ; p=.198)$, but the described result pattern was in the direction of a congruency effect. The main effect of order was not significant $(b=$ $-0.10 ; z=-0.51 ; p=.613)$. The main effect of task was not significant $(b=-0.26 ; z=$ $-1.38 ; p=.168)$. The three-way interaction of congruency, order, and task was significant $(b=-0.91 ; z=-2.71 ; p=.007)$, with a stronger congruency effect for the orientation task in the first half of the experiment but a stronger congruency effect for the motion task in the second half of the experiment. Finally, the four-way interaction of coherence, congruency, task, and order was significant $(b=1.58 ; z=3.43 ; p=.001)$ and was further explored in a post-hoc analysis (see below). None of the remaining interactions were significant.

The significant four-way interaction was further explored with two separate analyses which investigated the effect of coherence, congruency level, and order for each task (motion and orientation) separately. The same regression model was therefore fitted for each task, respectively and without the task variable as a fixed effect and random slope effect. Results of the motion task model revealed a significant main effect for coherence $(b=-6.53 ; z=-16.88 ; p<.001)$, with more erroneous responses on lower coherence levels. In addition, the results revealed a significant interaction between congruency and order $(b=1.20 ; z=2.41 ; p=.015)$, with an increased congruency effect on the second half of the experiment. Finally, results showed a significant three-way interaction $(b=-2.18 ; z=-3.12 ; p=.001)$, with a congruency effect on the 
first half of the experiment only for lower coherence levels and a congruency effect on the second half of the experiment for all coherence levels. Results of the orientation task model revealed a significant main effect for coherence $(b=-5.48 ; z=-15.65 ; p<.001)$, with more erroneous responses on lower coherence levels, but no other significant main effects or significant interactions.

\section{Analysis 2c: RTs as a Function of Distraction}

The results of this analysis are listed in Table 5. The main effect of distraction was significant $(b=-71.38 ; t=-6.44 ; p<.001)$, with slower responses on distractor trials compared to non-distractor trials. There was no main effect of task $(b=16.40 ; t$ $=1.47 ; p=.141)$ and no interaction between task and distractor $(b=6.87 ; t=0.62 ; p$ $=.535)$.

\section{Analysis 2d: Error Rates as a Function of Distraction}

The results of this analysis are listed in Table 6. The main effect of distraction was not significant $(b=-0.08 ; z=-1.60 ; p=.110)$ but the descriptive pattern of the results pointed towards more errors on distractor trials. There was no main effect of task $(b=0.04 ; z=0.83 ; p=.404)$ and no interaction between task and distractor $(b=$ $-0.02 ; z=-0.40 ; p=.687)$.

\section{Discussion}

The purpose of Experiment 2 was to replicate the result pattern of Experiment 1 with a different stimulus type, i.e., oriented leaves moving and orienting up or down on a naturalistic background. In line with Experiment 1, responses were slower and associated with higher error rates on lower coherence levels. In addition, participants were slower on incongruent compared to congruent trials and made marginally more errors on incongruent than congruent trials. A post-hoc analysis on a significant four-way interaction suggested that the congruency effect increased on the second half of the experiment for the motion task, indicated by higher error rates on more incongruent trials than on more congruent trials. However, please note that this four-way interaction 
has to be interpreted with caution, as our sample size was rather small, and the effect was not observed in the first experiment. Future studies may investigate the robustness of this interaction effect. The two tasks did not significantly differ with respect to RTs and error rates in Experiment 2. As in the previous experiment, distractor trials increased RTs and error rates were marginally increased on distractor trials. Together, these results mimic the result pattern of Experiment 1 and thus, we conclude that the results of Experiment 1 are independent of the object style and the background.

\section{General Discussion}

In this research project we developed and examined a ROK plugin for the jsPych software (de Leeuw, 2015). This ROK plugin has three major new features: (a) implementing objects instead of dots; (b) implementing several ROK layers on top of each other; and (c) implementing background pictures. We evaluated the quality of the data collection with two experiments (see Figure 2 which additionally tested experimental setups using the ROK plugin. In detail, we implemented oriented objects as stimuli so that participants can be instructed to respond according to the motion (motion detection task with two directions 'up' and 'down') or the orientation (orientation detection task with two directions 'up' and 'down') of the majority of the objects. With this experimental setup, the coherence of the target feature could be varied. In addition, the amount of overlapping or opposing feature directions could be varied, enabling different degrees of congruency. We applied different combinations of coherence and congruency levels and investigated the influence on participants' RTs and error rates with two experiments. The results of both experiments showed that lower coherence percentages increased participants' RTs and error rates. In addition, lower congruency levels (that is more incongruent stimuli) either increased participants' error rates (Experiment 1) or increased participants' RTs (Experiment 2), while the other performance measure (RTs in Experiment 1 and error rates in Experiment 2) revealed the same descriptive, but non-significant, pattern. Finally, we provide evidence that the implementation of an additional distractor stimulus layer led to increased RTs and error 
rates, compared to trials with no additional distractor layer. In the remainder of the discussion we further discuss each of the three new features in more detail.

\section{Implementing Oriented Objects}

Implementing oriented objects instead of dots enlarges the experimental possibilities of the random-object motion task. First, a second stimulus dimension enables researchers to investigate stimulus interference effects by varying congruency levels in addition to coherence levels. This integration of continuously varying stimulus interference allows researchers to apply this 'random-object motion task' in a similar way than other tasks applied in cognitive psychology designed to induce stimulus interference. Thus, the setting is similar to, for example, the Stroop task; on which participants are instructed to respond to the ink color of color words, with either overlapping (congruent stimuli; e.g., RED) color word meaning and color ink or non-overlapping color word meaning and color ink (incongruent stimuli; e.g., RED) (Cohen, Dunbar, \& Mcclelland, 1990; Stroop, 1935). Or it is similar to the flanker task, on which participants are instructed to respond to the direction of an arrow in the middle of a screen surrounded by arrows pointing in the same or opposing direction (Eriksen \& Eriksen, 1974). Yet, in contrast to these two traditional tasks, the difficulty of the random-object motion task as well as the congruency level can be varied continuously expanding the possibilities of task usage considerably.

\section{Implementing Several ROK Stimulus Layers at the Same Time}

Multiple ROKs may be presented at the same time, allowing the possibility to implement distraction stimuli of another irrelevant stimulus dimension. Results provided evidence that participants' performance decreased on trials with these additional distractor stimulus layers. This new feature may be especially relevant for experimental designs which need to induce distractor or even surprising stimuli (e.g. see Wessel, 2018; Wessel \& Aron, 2017). 


\section{Changing Object Visuals and Backgrounds}

Results of Experiment 2, in which more naturalistic visuals and a background were applied, revealed similar result patterns with regards to main effects of coherence and congruency level. Changing the aesthetic look, and with that the context of the stimulus layer of experiments, may be especially relevant for studies with younger cohorts, as children may understand the instructions better when responding to more aesthetic visuals such as upwards or downwards floating leaves in nature, as compared to upwards or downwards moving triangles on a black background. In addition, the option to change the background allows for an easy manipulation of contexts. This enables, for example, to consider context-specific adaptation to congruency levels (Braem et al. 2019); similar to the context-specific proportion congruency effect (Crump, Gong, \& Milliken, 2006, Crump, Vaquero, \& Milliken, 2008, Heinemann, Kunde, \& Kiesel, 2009, Schmidt \& Lemercier, 2019, Wendt \& Kiesel, 2011) or context-specific retrieval effects if target stimulus features, response features or other task parameters repeat or switch context-specifically (Dignath, Johannsen, Hommel, \& Kiesel, 2019; Dignath, Kiesel, Schiltenwolf, \& Hazeltine, 2021, Frings et al., 2020).

\section{Conclusion}

In sum, this ROK plugin serves as a platform for various research experiments in the fields of cognitive psychology or even developmental psychology, with gradual degrees of task difficulty and task interference, with the possibility to apply distractors, and with the possibility to implement different contexts. We hope that other researchers may benefit from this broad feature space integrated in the ROK plugin when designing experiments. 
References

Bates, D., Kliegl, R., Vasishth, S., \& Baayen, R. H. (2015). Parsimonious Mixed Models. Retrieved from https://arxiv.org/pdf/1506.04967.pdf

Bates, D., Mächler, M., Bolker, B., \& Walker, S. (2014). Fitting Linear Mixed-Effects Models using lme4. Journal of Statistical Software, 67(1), 51. Retrieved from http://arxiv.org/abs/1406.5823 doi: 10.18637/jss.v067.i01

Benetti, S., Zonca, J., Ferrari, A., Rezk, M., Rabini, G., \& Collignon, O. (2021). Visual motion processing recruits regions selective for auditory motion in early deaf individuals. NeuroImage, 230(August 2020), 117816. Retrieved from https://doi.org/10.1016/j.neuroimage.2021.117816 doi: 10.1016/j.neuroimage.2021.117816

Bhui, R. (2019). A statistical test for the optimality of deliberative time allocation. Psychonomic Bulletin and Review, 26(3), 855-867. doi: 10.3758/s13423-018-1555-1

Braem, S., Bugg, J. M., Schmidt, J. R., Crump, M. J., Weissman, D. H., Notebaert, W., \& Egner, T. (2019). Measuring Adaptive Control in Conflict Tasks. Trends in Cognitive Sciences, 23(9), 769-783. Retrieved from https://doi.org/10.1016/j.tics.2019.07.002 doi: 10.1016/j.tics.2019.07.002

Britten, K. H., Shadlen, M. N., Newsome, W. T., \& Movshon, J. a. (1992). The analysis of visual motion: a comparison of neuronal and psychophysical performance. The Journal of Neuroscience, 12(12), 4745-4765. Retrieved from http://eutils.ncbi.nlm.nih.gov/entrez/eutils/elink.fcgi?dbfrom= pubmed $\{\&\}$ id $=1464765\{\&\}$ retmode $=r e f\{\&\} \mathrm{cmd}=$ prlinks $\{\%\} 5$ Cnpapers $3: / /$ publication/uuid/E8CEBDDA-F6CF-4392-AEE5-5F9E687F3ED1 doi: 10.1.1.123.9899

Buhrmester, M., Kwang, T., \& Gosling, S. D. (2011). Amazon's mechanical Turk: A new source of inexpensive, yet high-quality, data? Perspectives on Psychological Science, 6(1), 3-5. doi: 10.1177/1745691610393980 
Cohen, J. D., Dunbar, K., \& Mcclelland, J. L. (1990). On the Control of Automatic Processes : A Parallel Distributed Processing Account of the Stroop Effect. Psychological Review, 97(3), 332-361.

Crump, M. J., Gong, Z., \& Milliken, B. (2006). The context-specific proportion congruent Stroop effect: Location as a contextual cue. Psychonomic Bulletin and Review, 13(2), 316-321. doi: 10.3758/BF03193850

Crump, M. J., McDonnell, J. V., \& Gureckis, T. M. (2013). Evaluating Amazon's Mechanical Turk as a Tool for Experimental Behavioral Research. PLoS ONE, 8(3). doi: 10.1371/journal.pone.0057410

Crump, M. J., Vaquero, J. M., \& Milliken, B. (2008). Context-specific learning and control: The roles of awareness, task relevance, and relative salience. Consciousness and Cognition, 17(1), 22-36. doi: 10.1016/j.concog.2007.01.004

Danielmeier, C., Eichele, T., Forstmann, B. U., Tittgemeyer, M., \& Ullsperger, M. (2011). Posterior medial frontal cortex activity predicts post-error adaptations in task-related visual and motor areas. Journal of Neuroscience, 31(5), 1780-1789. doi: 10.1523/JNEUROSCI.4299-10.2011

de Leeuw, J. R. (2015). jsPsych: A JavaScript library for creating behavioral experiments in a Web browser. Behavior Research Methods, 47(1), 1-12. doi: 10.3758/s13428-014-0458-y

Dignath, D., Johannsen, L., Hommel, B., \& Kiesel, A. (2019). Reconciling cognitive-control and episodic-retrieval accounts of sequential conflict modulation: Binding of control-states into event-files. Journal of Experimental Psychology: Human Perception and Performance, 45 (9), 1265-1270. doi: 10.1037/xhp0000673

Dignath, D., Kiesel, A., Schiltenwolf, M., \& Hazeltine, E. (2021). Multiple Routes to Control in the Prime-Target Task: Congruence Sequence Effects Emerge Due to Modulation of Irrelevant Prime Activity and Utilization of Temporal Order Information. Journal of Cognition, 4(1), 1-19. doi: 10.5334/joc.143

Eriksen, B., \& Eriksen, C. (1974). Effects of noise letters upon the identification of a 
target letter in a nonsearch task. Perception and Psychophysics, 16(1), 143-149.

Faul, F., Erdfelder, E., Lang, A.-G., \& Buchner, A. (2007). G*Power 3: A flexible statistical power analysis program for the social, behavioral, and biomedical sciences FRANZ. Journal of Materials and Environmental Science, 39(2), $175-191$.

Frings, C., Hommel, B., Koch, I., Rothermund, K., Dignath, D., Giesen, C., .. Philipp, A. (2020). Binding and Retrieval in Action Control ( BRAC ). Trends in Cognitive Sciences, 24(5), 375-387. doi: 10.1016/j.tics.2020.02.004

Guterstam, A., \& Graziano, M. S. (2020). Implied motion as a possible mechanism for encoding other people's attention. Progress in Neurobiology, 190 (March), 101797. Retrieved from https://doi.org/10.1016/j.pneurobio.2020.101797 doi: 10.1016/j.pneurobio.2020.101797

Guterstam, A., Wilterson, A. I., Wachtell, D., \& Graziano, M. S. (2020). Other people's gaze encoded as implied motion in the human brain. Proceedings of the National Academy of Sciences of the United States of America, 117(23), 13162-13167. doi: $10.1073 /$ pnas. 2003110117

Heinemann, A., Kunde, W., \& Kiesel, A. (2009). Context-specific prime-congruency effects: On the role of conscious stimulus representations for cognitive control. Consciousness and Cognition, 18(4), 966-976. Retrieved from http://dx.doi.org/10.1016/j.concog.2009.08.009 doi: 10.1016/j.concog.2009.08.009

Judd, C. M., Westfall, J., \& Kenny, D. A. (2017). Experiments with More Than One Random Factor: Designs, Analytic Models, and Statistical Power. Annual Review of Psychology, 68(October 2016), 601-625. doi: 10.1146/annurev-psych-122414-033702

Kang, Y. H., Löffler, A., Jeurissen, D., Zylberberg, A., Wolpert, D. M., \& Shadlen, M. N. (2021). Multiple decisions about one object involve parallel sensory acquisition but time-multiplexed evidence incorporation. eLife, 10, 1-44. doi: 10.7554/eLife.63721 
Kayser, A. S., Erickson, D. T., Buchsbaum, B. R., \& D'Esposito, M. (2010). Neural representations of relevant and irrelevant features in perceptual decision making. Journal of Neuroscience, 30(47), 15778-15789. doi: 10.1523/JNEUROSCI.3163-10.2010

Krueger, P. M., van Vugt, M. K., Simen, P., Nystrom, L., Holmes, P., \& Cohen, J. D. (2017). Evidence accumulation detected in BOLD signal using slow perceptual decision making. Journal of Neuroscience Methods, 281, 21-32. Retrieved from http://dx.doi.org/10.1016/j.jneumeth.2017.01.012 doi:

10.1016/j.jneumeth.2017.01.012

Krzeminski, D., \& Zhang, J. (2021). Imperfect Integration: Sensory Congruency Between Multiple Sources Modulates Selective Decision-Making Processes.

Kuznetsova, A., Brockhoff, P. B., \& Christensen, R. H. B. (2017). lmerTest package: Tests in linear mixed effects models. Journal of Statistical Software, 82(13), 1-26.

Lüdecke, D. (2020). sjPlot: Data Visualization for Statistics in Social Science.

Retrieved from https://cran.r-project.org/package=sjPlot doi: 10.5281/zenodo. 1308157

Mante, V., Sussillo, D., Shenoy, K. V., \& Newsome, W. T. (2013). Context-dependent computation by recurrent dynamics in prefrontal cortex. Nature, 503, 78-84.

Retrieved from http://dx.doi.org/10.1038/nature12742 doi:

$10.1038 /$ nature 12742

Marques, T., Summers, M. T., Fioreze, G., Fridman, M., Dias, R. F., Feller, M. B., \& Petreanu, L. (2018). A Role for Mouse Primary Visual Cortex in Motion Perception. Current Biology, 28(11), 1703-1713.e6. Retrieved from https://doi.org/10.1016/j.cub.2018.04.012 doi: 10.1016/j.cub.2018.04.012

Musslick, S., Bizyaeva, A., Agaron, S., Leonard, N., \& Cohen, J. D. (2019).

Stability-Flexibility Dilemma in Cognitive Control:A Dynamical System Perspective. Proceedings of the 41st Annual Meeting of the Cognitive Science Society(August), pp. 2420-2426. 
Purcell, B. A., \& Kiani, R. (2016). Hierarchical decision processes that operate over distinct timescales underlie choice and changes in strategy. Proceedings of the National Academy of Sciences of the United States of America, 113(31), E4531-E4540. doi: 10.1073/pnas.1524685113

Quené, H. (2008). Examples of mixed-effects modelling. , 1-40.

Quené, H., \& Van Den Bergh, H. (2004). On multi-level modeling of data from repeated measures designs: A tutorial. Speech Communication, 43(1-2), 103-121. doi: 10.1016/j.specom.2004.02.004

R Core Team. (2013). R: A Language and Environment for Statistical Computing.

Vienna, Austria. Retrieved from http://www.r-project.org/

Rajananda, S., Lau, H., \& Odegaard, B. (2018). A Random-Dot Kinematogram for Web-Based Vision Research. Journal of Open Research Software, 6(6). doi: https://doi.org/10.5334/jors.194

Ramsey, S. R., Thompson, K. L., McKenzie, M., \& Rosenbaum, A. (2016).

Psychological research in the internet age: The quality of web-based data. Computers in Human Behavior, 58, 354-360. Retrieved from http://dx.doi.org/10.1016/j.chb.2015.12.049 doi:

10.1016/j.chb.2015.12.049

Ritz, H., \& Shenhav, A. (2019). Parametric control of distractor-oriented attention. In Proceedings of the 41st annual meeting of the cognitive science society (pp. 967-973).

Schmidt, J. R., \& Lemercier, C. (2019). Context-specific proportion congruent effects: Compound-cue contingency learning in disguise. Quarterly Journal of Experimental Psychology, 72(5), 1119-1130. doi: 10.1177/1747021818787155

Shadlen, M. N., Britten, K. H., Newsome, W. T., \& Movshon, J. A. (1996). Computational Analysis:Y2K. Journal of Neuroscience, 16(4), 1486-1510. doi: $10.1126 /$ science.286.5448.2281

Shenhav, A., Straccia, M. A., Musslick, S., Botvinick, M. M., \& Cohen, J. D. (2018). Dissociable neural mechanisms track evidence accumulation for selection of 
attention versus action. Nature Communications(9), 2485. Retrieved from http://dx.doi.org/10.1038/s41467-018-04841-1 doi:

$10.1038 / \mathrm{s} 41467-018-04841-1$

Spitzer, M. W. H., Kiesel, A., \& Dignath, D. (2021). Performance Errors Influence Voluntary Task Choices. , 1-41. doi: 10.17605/OSF.IO/FZD9V

Spitzer, M. W. H., Musslick, S., Shvartsman, M., Shenhav, A., \& Cohen, J. D. (2019). Asymmetric switch costs as a function of task strength. In Proceedings of the 41th annual conference of the Cognitive Science Society. Montreal, CA.

Steyvers, M., Hawkins, G. E., Karayanidis, F., \& Brown, S. D. (2019). A large-scale analysis of task switching practice effects across the lifespan. Proceedings of the National Academy of Sciences of the United States of America, 116(36), 17735-17740. doi: 10.1073/pnas.1906788116

Stroop, J. R. (1935). Studies of interference in serial verbal reactions. Journal of experimental psychology., 18(6), 643-662.

von Lautz, A., Herding, J., \& Blankenburg, F. (2019). Neuronal signatures of a random-dot motion comparison task. NeuroImage, 193, 57-66. Retrieved from https://doi.org/10.1016/j.neuroimage.2019.02.071 doi:

10.1016/j.neuroimage.2019.02.071

Wendt, M., \& Kiesel, A. (2011). Conflict adaptation in time: Foreperiods as contextual cues for attentional adjustment. Psychonomic Bulletin and Review, 18(5), 910-916. doi: 10.3758/s13423-011-0119-4

Wessel, J. R. (2018). An adaptive orienting theory of error processing. Psychophysiology, 55, 1-21. doi: 10.1111/psyp.13041

Wessel, J. R., \& Aron, A. R. (2017). On the Globality of Motor Suppression: Unexpected Events and Their Influence on Behavior and Cognition. Neuron, 93(2), 259-280. Retrieved from http://dx.doi.org/10.1016/j.neuron.2016.12.013 doi: 10.1016/j.neuron.2016.12.013 


\section{Table 1}

\section{Parameters and their Description for the ROK Plugin.}

\begin{tabular}{|c|c|}
\hline Parameter & Description (ROK) \\
\hline choices & The valid keys that the subject can press to indicate a response. \\
\hline correct_choice & The correct keys for that trial. \\
\hline trial_duration & The length of stimulus presentation. Zero for endless loop. \\
\hline response_ends_trial & If true, then any valid key will end the trial. \\
\hline number_of_apertures & Number of apertures. If greater then one, other parameters of trial should be arrays. \\
\hline density_unit_area & If this parameter is set, number_of_objects is interpreted as number_of_objects per density_unit_area (in pixels*pixels) \\
\hline number_of_oobs & The number of oriented objects per set in the stimulus. \\
\hline coherence & The proportion of dots that move together in the coherent direction. Range is 0 to 1 . \\
\hline opposite_coherence & The proportion of moving in the direction opposite of the coherent direction. Range is 0 to (1-coherence). \\
\hline coherent_movement_direction & The direction of coherent motion in degrees $(0$ degree $=$ right $)$. \\
\hline coherent_orientation & The orientation of the objects in degree $(0$ degree $=$ right $)$. \\
\hline coherence_movement & The percentage of oriented objects moving in the coherent direction. \\
\hline coherence_orientation & The percentage of objects that are oriented in the coherent orientation. \\
\hline coherence_movement_opposite & The percentage of oriented objects moving in the direction opposite of the coherent direction. \\
\hline coherence_orientation_opposite & The percentage of objects that are oriented opposite of the coherent orientation. \\
\hline movement_speed & The movement speed of the oobs in (percentage of aperature_width)/second. \\
\hline movement_speed_randomisation & $\begin{array}{l}\text { The percentage of randomisation in movement speed; } 0=\text { all orientated objects move with defined } \\
\text { speed in movement_speed; } 100=\text { movement speeds from } 0 \text { to } 2 \mathrm{x} \text { movement_speed. }\end{array}$ \\
\hline random_movement_type & Type of random movement: 0 direction is random but fixed; 1 movement direction of incoherent oobs changes over time. \\
\hline random_orientation_type & Type of random movement; 0 - orientation is random but fixed, 1 - orientation of incoherent oobs changes over time. \\
\hline oob_size & The size of the oriented objects in percentage of aperture_width. \\
\hline oob_color & The color of the oobs. \\
\hline background_color & The color of the background. \\
\hline background_image & Background image, can be. \\
\hline aperture_width & The width of the aperture in pixels. \\
\hline aperture_height & he height of the aperture in pixels. \\
\hline aperture_position_left & Position of midpoint of aperture in $\mathrm{x}$ direction in percentage of window width ( 0 being left, 100 being right). \\
\hline aperture_position_top & Position of midpoint of aperture in y direction in percentage of window width ( 0 being top, 100 being bottom). \\
\hline aperture_shape & 0 - rectangular, 1 - elliptic. \\
\hline stimulus_type & Appearance of stimulus (0-triangles, 1-circle,2-square,3-bird, 4-image). \\
\hline stimulus_image & Pictures of stimuli, can be key-framed (animated) or randomised, see documentation inside the plugin code. \\
\hline stimulus_image_keyframes & Number of keyframes in stimulus images. \\
\hline stimulus_keyframe_time & Time between keyframes in seconds. \\
\hline stimulus_mirror & $\begin{array}{l}\text { Mirror image instead of rotating ( } 1 \text { - x axis, } 2 \text { - y axis). } \\
\text { Can be useful for oobs that have two orientation axis (e.g front to back and up and down). }\end{array}$ \\
\hline prompt & Prompt that is presented above the stimulus. \\
\hline fade_out & Fade the oobs on the edges of the aperture. \\
\hline experiment_congruency_mode & $\begin{array}{l}\text { Sets experiment to congruency mode, experiment_main_task has to be set }(0=\text { movement or } 1=\text { orientation) } \\
\text { if this is set to } 1 \text { or } 2 \text {. The congruency of the task does only apply to coherent oobs of the main task. } \\
\text { If this is set to } 1 \text { the remaining oobs secondary feature (the non-task feature) is set at random. } \\
\text { If this is set to } 2 \text { the remaining oobs have the same movement and orientation direction. }\end{array}$ \\
\hline experiment_main_task & $\begin{array}{l}\text { Sets the main task when experiment is in congruency mode. The congruency of the other task then only applies } \\
\text { to non random oobs of main task ( } 0 \text { - movement task, } 1 \text { - orientation task). }\end{array}$ \\
\hline units & Units in which size and speed of oobs is expressed (null - percentage of aperture width, px - pixels. \\
\hline
\end{tabular}


Table 2

The plugin collects all parameter data of Table 1 and the following data for each trial

\begin{tabular}{|l|l|}
\hline Parameter & Description (ROK) \\
\hline rt & The response time in ms for the subject to make a response. \\
\hline key_press & The key that the subject pressed. The value corresponds to the Javascript Char Code (Key Code). \\
\hline correct & Whether or not the subject's key press corresponded to those provided in correct_choice. \\
\hline frame_rate & The average frame rate for the trial. 0 denotes that the subject responded before the appearance of the second frame. \\
\hline number_of_frames & The number of frames that was shown in this trial. \\
\hline frame_rate_array & The array that holds the number of milliseconds for each frame in this trial. \\
\hline canvas_width & The width of the canvas in pixels. \\
\hline canvas_hight & The height of the canvas in pixels. \\
\hline
\end{tabular}




\section{Table 3}

Estimates (Betas), 95\% Confidence Intervals, $t$-Values, and $p$-Values of the Linear Mixed Model of Analysis 1a (Experiment 1) and 2a (Experiment 2).

\begin{tabular}{|c|c|c|c|c|c|c|c|c|}
\hline \multirow[b]{2}{*}{ Coeffcient } & \multicolumn{4}{|c|}{ Exp 1: RT } & \multicolumn{4}{|c|}{$\operatorname{Exp} 2: \mathrm{RT}$} \\
\hline & Estimates & Conf. Int (95\%) & t-Value & p-Value & Estimates & Conf. Int (95\%) & t-Value & $p$-Value \\
\hline Intercept & 1306.22 & $1205.03-1407.42$ & 25.30 & $<0.001$ & 1267.31 & $1153.74-1380.88$ & 21.87 & $<0.001$ \\
\hline Coherence & -433.96 & $-551.82--316.11$ & -7.22 & $<0.001$ & -412.69 & $-506.57--318.80$ & -8.62 & $<0.001$ \\
\hline Congruency & -51.26 & $-131.00-28.48$ & -1.26 & 0.208 & -95.76 & $-166.80--24.72$ & -2.64 & 0.008 \\
\hline Order & -47.86 & $-106.98-11.26$ & -1.59 & 0.113 & 2.17 & $-52.87-57.22$ & 0.08 & 0.938 \\
\hline Task & 85.49 & $26.37-144.62$ & 2.83 & 0.005 & 21.19 & $-33.85-76.24$ & 0.75 & 0.451 \\
\hline Coherence:Congruency & -14.99 & $-116.35-86.38$ & -0.29 & 0.772 & 30.33 & $-60.44-121.11$ & 0.65 & 0.513 \\
\hline Coherence:Order & 50.98 & $-16.12-118.09$ & 1.49 & 0.136 & -29.94 & $-89.57-29.69$ & -0.98 & 0.325 \\
\hline Congruency:Order & 39.56 & $-39.30-118.43$ & 0.98 & 0.326 & 5.82 & $-64.66-76.30$ & 0.16 & 0.871 \\
\hline Coherence:Task & -135.43 & $-202.53--68.33$ & -3.96 & $<0.001$ & 52.13 & $-7.50-111.76$ & 1.71 & 0.087 \\
\hline Congruency:Task & 88.95 & $10.09-167.82$ & 2.21 & 0.027 & 60.46 & $-10.02-130.94$ & 1.68 & 0.093 \\
\hline Order:Task & -55.83 & $-157.02-45.37$ & -1.08 & 0.280 & -65.90 & $-179.48-47.67$ & -1.14 & 0.255 \\
\hline Coherence:Congruency:Order & -76.69 & $-178.01-24.64$ & -1.48 & 0.138 & -21.47 & $-112.21-69.27$ & -0.46 & 0.643 \\
\hline Coherence:Congruency:Task & -81.53 & $-182.85-19.80$ & -1.58 & 0.115 & -81.09 & $-171.83-9.65$ & -1.75 & 0.080 \\
\hline Coherence:Order:Task & 50.19 & $-67.66-168.05$ & 0.83 & 0.404 & 66.79 & $-27.09-160.67$ & 1.39 & 0.163 \\
\hline Congruency:Order:Task & -58.62 & $-138.36-21.12$ & -1.44 & 0.150 & 50.56 & $-20.48-121.60$ & 1.39 & 0.163 \\
\hline Coherence:Congruency:Order:Task & 82.98 & $-18.38-184.34$ & 1.60 & 0.109 & -75.38 & $-166.16-15.39$ & -1.63 & 0.104 \\
\hline \multicolumn{9}{|l|}{ Random Effects } \\
\hline$\sigma^{2}$ & \multicolumn{4}{|l|}{66673.24} & \multicolumn{4}{|l|}{59350.35} \\
\hline $\mathrm{\tau}_{00}$ & \multicolumn{4}{|c|}{61955.67 subject_id } & \multicolumn{4}{|c|}{94580.53 subject_id } \\
\hline \multirow[t]{3}{*}{$\mathrm{\tau}_{11}$} & \multicolumn{4}{|c|}{77678.06 subject_id.coherence } & \multicolumn{4}{|c|}{46192.15 subject_id.coherence } \\
\hline & \multicolumn{4}{|c|}{1106.42 subject_id.congruency } & \multicolumn{4}{|c|}{677.44 subject_id.congruency } \\
\hline & \multicolumn{4}{|c|}{6123.98 subject_id.task1 } & \multicolumn{4}{|c|}{7636.41 subject_id.task1 } \\
\hline \multirow[t]{3}{*}{$\rho_{01}$} & \multicolumn{4}{|l|}{-0.90} & \multicolumn{4}{|l|}{-0.94} \\
\hline & \multicolumn{4}{|l|}{-0.37} & \multicolumn{4}{|l|}{-0.05} \\
\hline & \multicolumn{4}{|l|}{0.24} & \multicolumn{4}{|l|}{0.13} \\
\hline Observations & \multicolumn{4}{|l|}{11172} & \multicolumn{4}{|l|}{12363} \\
\hline
\end{tabular}

Note. Sigma squared denotes the residual variance of the random effects. Tau00 indicates the variance between participants. Tau 11 indicates the variance between participants on random slopes for each random effect. Rho indicates the correlations between participants' random intercept and their random slope, respectively. 


\section{Table 4}

Log-Odds (Betas), 95\% Confidence Intervals, $t$-Values, and p-Values of the Logistic Mixed Model of Analysis $1 b$ (Experiment 1) and 2b (Experiment 2).

\begin{tabular}{|c|c|c|c|c|c|c|c|c|}
\hline \multirow[b]{2}{*}{ Coeffcient } & \multicolumn{4}{|c|}{ Exp 1: Error Rate } & \multicolumn{4}{|c|}{ Exp 2: Error Rate } \\
\hline & Log-Odds & s Conf. Int (95\%) & z-Value & p-Value & Log-Odds & Conf. Int (95\%) & z-Value & $p$-Value \\
\hline Intercept & 3.45 & $3.07-3.83$ & 17.67 & $<0.001$ & 3.25 & $2.84-3.66$ & 15.64 & $<\mathbf{0 . 0 0 1}$ \\
\hline Coherence & -5.95 & $-6.44--5.45$ & -23.72 & $<\mathbf{0 . 0 0 1}$ & -5.93 & $-6.44--5.42$ & -22.92 & $<0.001$ \\
\hline Congruency & -1.11 & $-1.73--0.49$ & -3.50 & $<0.001$ & -0.43 & $-1.09-0.23$ & -1.29 & 0.198 \\
\hline Order & 0.10 & $-0.27-0.46$ & 0.52 & 0.603 & -0.10 & $-0.47-0.28$ & -0.51 & 0.613 \\
\hline Task & 0.32 & $-0.05-0.69$ & 1.72 & 0.085 & -0.27 & $-0.64-0.11$ & -1.39 & 0.164 \\
\hline Coherence:Congruency & 0.23 & $-0.63-1.08$ & 0.52 & 0.602 & -0.64 & $-1.54-0.27$ & -1.38 & 0.168 \\
\hline Coherence:Order & -0.03 & $-0.52-0.46$ & -0.10 & 0.917 & 0.24 & $-0.26-0.75$ & 0.95 & 0.343 \\
\hline Congruency:Order & -0.36 & $-0.97-0.25$ & -1.16 & 0.248 & 0.35 & $-0.29-0.98$ & 1.07 & 0.284 \\
\hline Coherence:Task & -0.60 & $-1.09--0.11$ & -2.41 & 0.016 & 0.49 & $-0.02-0.99$ & 1.88 & 0.060 \\
\hline Congruency:Task & 0.52 & $-0.08-1.13$ & 1.69 & 0.091 & 0.12 & $-0.51-0.76$ & 0.38 & 0.705 \\
\hline Order:Task & -0.34 & $-0.73-0.04$ & -1.76 & 0.079 & 0.18 & $-0.22-0.59$ & 0.88 & 0.377 \\
\hline Coherence:Congruency:Order & 0.36 & $-0.49-1.21$ & 0.83 & 0.406 & -0.67 & $-1.57-0.24$ & -1.45 & 0.147 \\
\hline Coherence:Congruency:Task & -0.40 & $-1.26-0.45$ & -0.93 & 0.352 & 0.02 & $-0.88-0.92$ & 0.05 & 0.962 \\
\hline Coherence:Order:Task & 0.51 & $0.02-1.00$ & 2.03 & 0.042 & -0.34 & $-0.85-0.17$ & -1.31 & 0.190 \\
\hline Congruency:Order:Task & 0.24 & $-0.38-0.86$ & 0.77 & 0.444 & -0.91 & $-1.56--0.25$ & -2.71 & 0.007 \\
\hline Coherence:Congruency:Order:Task & -0.19 & $-1.04-0.66$ & -0.44 & 0.662 & 1.58 & $0.68-2.48$ & 3.43 & 0.001 \\
\hline \multicolumn{9}{|l|}{ Random Effects } \\
\hline$\sigma^{2}$ & \multicolumn{4}{|l|}{3.29} & \multicolumn{4}{|l|}{3.29} \\
\hline$\tau_{00}$ & \multicolumn{4}{|c|}{0.17 subject_id } & \multicolumn{4}{|c|}{0.29 subject_id } \\
\hline \multirow[t]{2}{*}{$\mathrm{T}_{11}$} & \multicolumn{4}{|c|}{0.13 subject_id.congruency } & \multicolumn{4}{|c|}{0.24 subject_id.congruency } \\
\hline & \multicolumn{4}{|c|}{0.08 subject_id.task1 } & \multicolumn{4}{|c|}{0.06 subject_id.task1 } \\
\hline \multirow[t]{2}{*}{$\rho_{01}$} & \multicolumn{4}{|l|}{-0.10} & \multicolumn{4}{|l|}{-0.79} \\
\hline & \multicolumn{4}{|l|}{-0.71} & \multicolumn{4}{|l|}{-0.65} \\
\hline Observations & \multicolumn{4}{|l|}{18225} & \multicolumn{4}{|l|}{18801} \\
\hline
\end{tabular}

Note. Sigma squared denotes the residual variance of the random effects. Tau00 indicates the variance between participants. Tau 11 indicates the variance between participants on random slopes for each random effect. Rho indicates the correlations between participants' random intercept and their random slope, respectively. 


\section{Table 5}

Estimates (Betas), 95\% Confidence Intervals, $t$-Values, and $p$-Values of the Linear Mixed Model of Analysis 1c (Experiment 1) and 2c (Experiment 2).

\begin{tabular}{|c|c|c|c|c|c|c|c|c|}
\hline \multirow[b]{2}{*}{ Coeffcient } & \multicolumn{4}{|c|}{ Exp 1: RT } & \multicolumn{4}{|c|}{ Exp 2: RT } \\
\hline & Estimates & Conf. Int (95\%) & t-Value & p-Value & Estimates & $\begin{array}{c}\text { Conf. Int } \\
(95 \%)\end{array}$ & t-Value & $p$-Value \\
\hline Intercept & 1226.03 & $\begin{array}{c}1177.48- \\
1274.58\end{array}$ & 49.50 & $<0.001$ & 1176.11 & $\begin{array}{c}1096.17- \\
1256.06\end{array}$ & 28.83 & $<0.001$ \\
\hline Distractor & -94.74 & $\begin{array}{c}-116.65- \\
-72.84\end{array}$ & -8.48 & $<0.001$ & -71.38 & $\begin{array}{c}-93.10- \\
-49.66\end{array}$ & -6.44 & $<0.001$ \\
\hline Task & -23.34 & $-45.47--1.21$ & -2.07 & 0.039 & 16.40 & $-5.43-38.23$ & 1.47 & 0.141 \\
\hline Distractor:Task & 39.00 & $17.08-60.92$ & 3.49 & $<0.001$ & 6.87 & $\begin{array}{c}-14.87- \\
28.62\end{array}$ & 0.62 & 0.535 \\
\hline \multicolumn{9}{|l|}{ Random Effects } \\
\hline$\sigma^{2}$ & \multicolumn{4}{|l|}{80177.57} & \multicolumn{4}{|l|}{77353.51} \\
\hline$\tau_{00}$ & \multicolumn{4}{|c|}{15564.72 subject_id } & \multicolumn{4}{|c|}{52276.72 subject_id } \\
\hline Observations & 1946 & & & & 2028 & & & \\
\hline
\end{tabular}

Note. Sigma squared denotes the residual variance of the random effects. Tau00 indicates the variance between participants. 
Table 6

Log-Odds (Betas), 95\% Confidence Intervals, $t$-Values, and p-Values of the Logistic

Mixed Model of Analysis 1d (Experiment 1) and 2d (Experiment 2).

\begin{tabular}{|c|c|c|c|c|c|c|c|c|}
\hline \multirow[b]{2}{*}{ Coeffcient } & \multicolumn{4}{|c|}{ Exp 1: Error Rate } & \multicolumn{4}{|c|}{ Exp 2: Error Rate } \\
\hline & Log-Odds & s Conf. Int (95\% & z-Value & $p$-Value & Log-Odds & s Conf. Int (95\%, & z-Value & $p$-Value \\
\hline Intercept & -0.55 & $-0.70--0.41$ & -7.54 & $<0.001$ & -0.54 & $-0.69--0.40$ & -7.40 & $<0.001$ \\
\hline Distractor & -0.07 & $-0.17-0.04$ & -1.24 & 0.215 & -0.08 & $-0.18-0.02$ & -1.60 & 0.110 \\
\hline Task & -0.11 & $-0.21--0.01$ & -2.08 & 0.038 & 0.04 & $-0.06-0.14$ & 0.83 & 0.404 \\
\hline Distractor:Task & 0.13 & $0.03-0.23$ & 2.47 & 0.014 & -0.02 & $-0.12-0.08$ & -0.40 & 0.687 \\
\hline \multicolumn{9}{|l|}{ Random Effects } \\
\hline$\sigma^{2}$ & \multicolumn{4}{|l|}{3.29} & \multicolumn{4}{|l|}{3.29} \\
\hline $\mathrm{\tau}_{00}$ & \multicolumn{4}{|c|}{0.08 subject_id } & \multicolumn{4}{|c|}{0.09 subject_id } \\
\hline Observations & 4507 & & & & 4732 & & & \\
\hline
\end{tabular}

Note. Sigma squared denotes the residual variance of the random effects. Tau00 indicates the variance between participants. 


\section{Figure 1}

Experiment Stimuli for Experiment 1 (Left Panel; Oriented Isosceles Triangle Objects) and Experiment 2 (Right Panel; Oriented Leaf Objects)
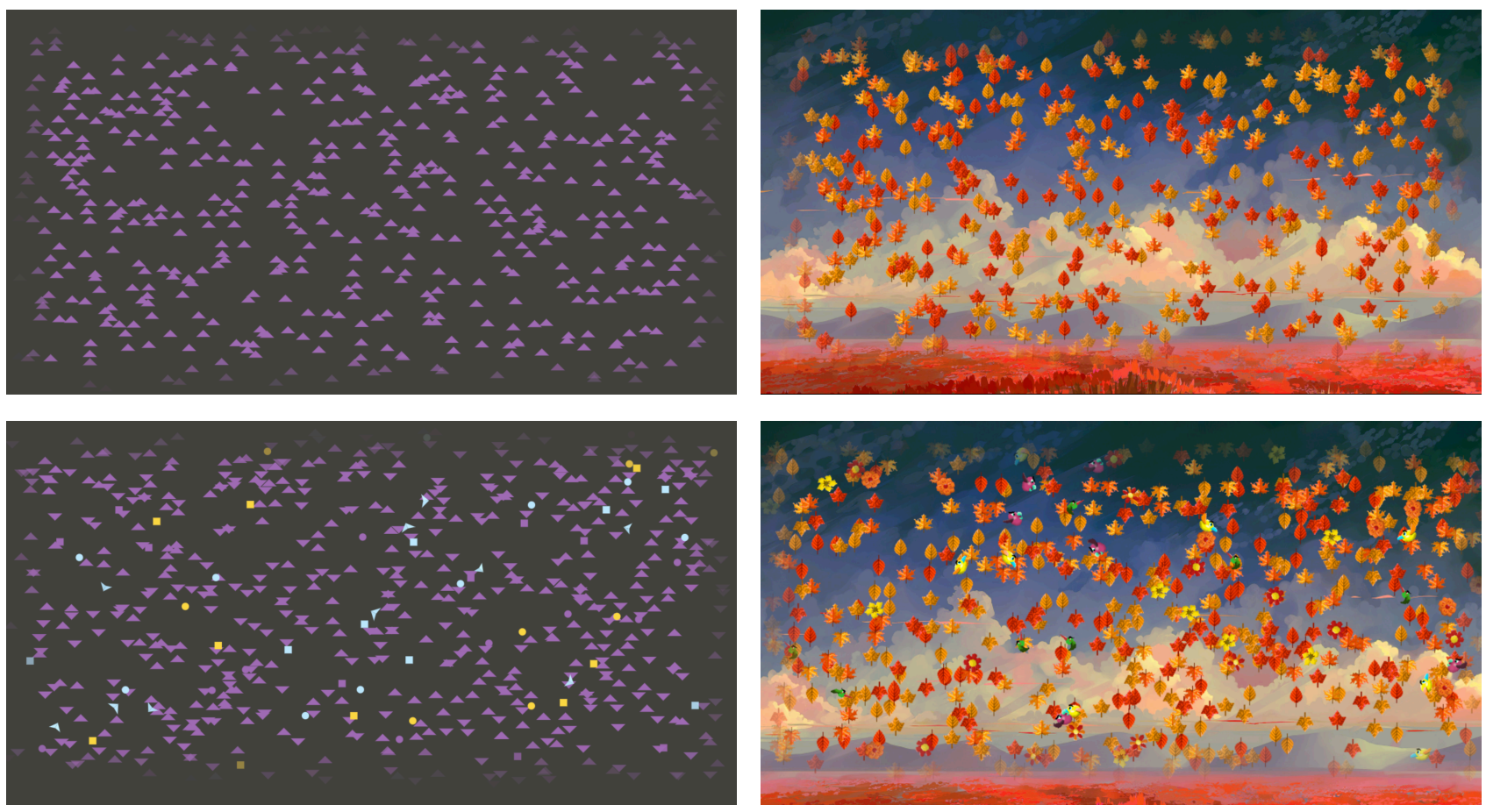

Note. In both tasks, participants were instructed to respond to the coherent moving direction (up or down) or the coherent orientation direction (up or down). The upper row depicts the target stimulus layer, for each task respectively. The bottom row shows the target and distractor stimulus layer presented at the same time, for each task respectively. 


\section{Figure 2}

Data Collection Quality for Different Internet Browser.

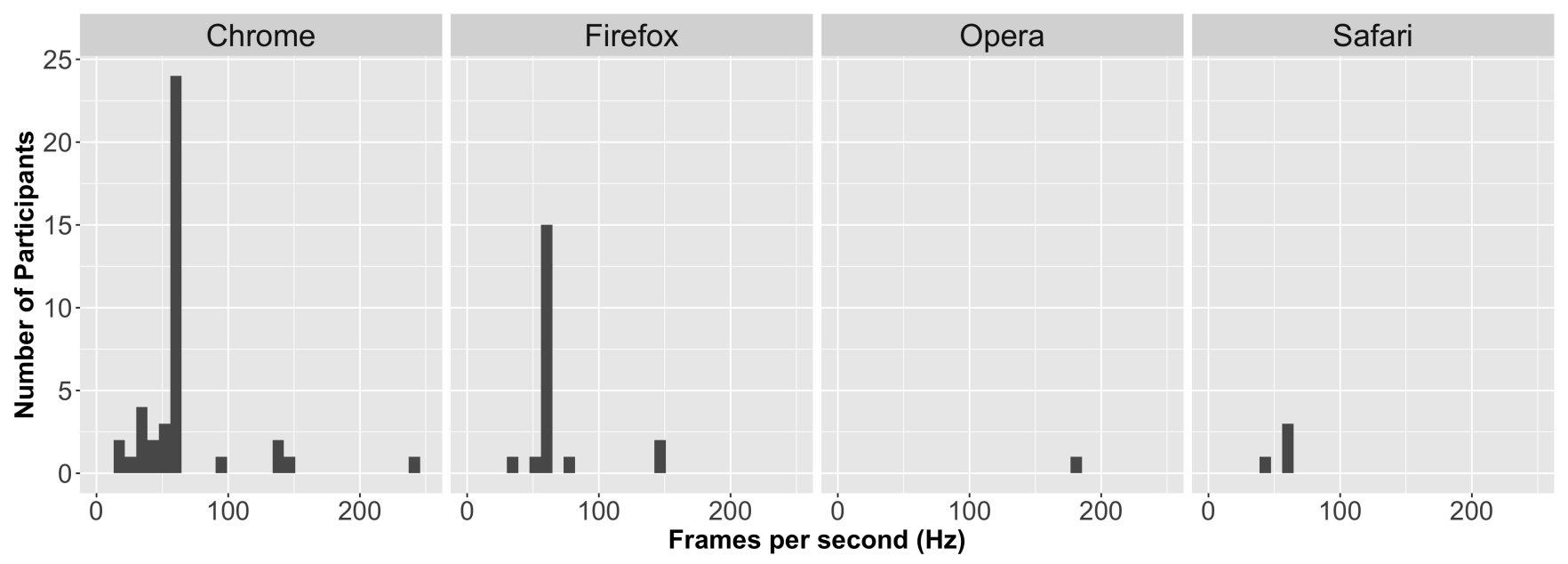

Note. The average frame per second $(\mathrm{Hz})$ for each participant for both experiments was computed and plotted in a histogram for the browser the participant used. Most participants used Chrome or Firefox while only few participants used Opera or Safari. 


\section{Figure 3}

RTs and Error Rates as a Function of Coherence and Congruency Level for each Experiment, Respectively.
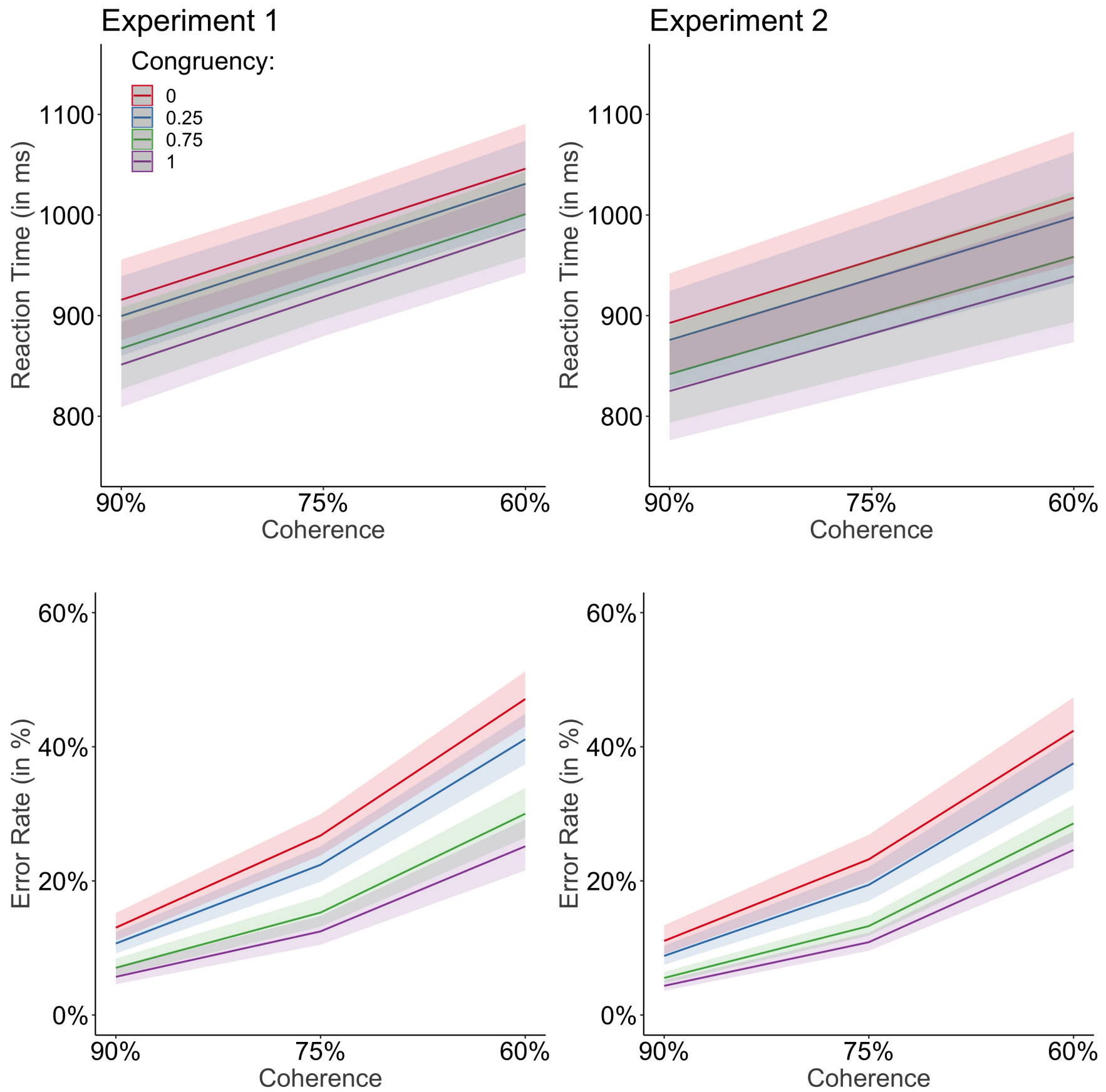

Note. RTs and error rates increased with decreasing coherence percentages and lower congruency levels. The results of Experiment 2 mimick the results of Experiment 1. 